\title{
Chondroprotective Effects and Multitarget Mechanisms of Fu Yuan Capsule in a Rat Osteoarthritis Model
}

\author{
Li Zeng, ${ }^{1,2}$ Cai Zhi Xiao, ${ }^{2,3}$ Zi Ting Deng, ${ }^{1,2,4}$ and Rong Heng Li ${ }^{1,2}$ \\ ${ }^{1}$ Department of Combination of Chinese and Western Medicine, The First Affiliated Hospital of Chongqing Medical University, \\ Chongqing 400016, China \\ ${ }^{2}$ Laboratory Research Center, The First Affiliated Hospital of Chongqing Medical University, Chongqing 400016, China \\ ${ }^{3}$ Department of Traditional Chinese Medicine for Tumor, Chongqing Cancer Institute, Chongqing 400030, China \\ ${ }^{4}$ Department of Rehabilitation Medicine, The Affiliated Rehabilitation Hospital of Chongqing Medical University, \\ Chongqing 400016, China
}

Correspondence should be addressed to Rong Heng Li; baihe_han@126.com

Received 12 October 2016; Revised 23 November 2016; Accepted 24 November 2016; Published 11 January 2017

Academic Editor: Antonella Fioravanti

Copyright ( 2017 Li Zeng et al. This is an open access article distributed under the Creative Commons Attribution License, which permits unrestricted use, distribution, and reproduction in any medium, provided the original work is properly cited.

Fu Yuan Capsule (FYC) has been clinically used for osteoarthritis (OA) and its related diseases for many years in China. However, its pharmacological mechanism remains unclear. This study aimed to investigate the potential chondroprotective effects of FYC on articular cartilage. Rat OA model was induced by anterior cruciate ligament transection. A group of rats was treated with FYC for 12 weeks. Joint structure, types I and II collagen, and proteoglycan were evaluated by histological examination. The expression of C-terminal crosslinking telopeptide of type II collagen, hydroxyproline, a disintegrin and metalloproteinase with thrombospondin motifs, matrix metalloproteinase, interleukin-1 beta, nitric oxide, prostaglandin E2, heat-shock protein 70 , transforming growth factor-beta, osteoprotegerin, and receptor activator of nuclear factor $\kappa \mathrm{B}$ ligand were detected. Treatment with FYC could protect against articular cartilage injury. FYC treatment significantly decreased the extracellular matrix degradation factors and inflammatory mediators. Moreover, articular cartilage protective factors were increased in the FYC group. The current finding suggests that FYC protects articular cartilage in a rat OA model through various ways. Thus, it may be an effective agent for OA treatment.

\section{Introduction}

Osteoarthritis (OA) has become a significant clinical problem worldwide and is expected to worsen with the growth of the aging population. Existing drug treatments include nonsteroidal anti-inflammatory drugs and selective cyclooxygenase-2 inhibitors. However, pharmacological interventions do not exceed beyond addressing chronic pain and fail to prevent cartilage damage and the associated destruction of other joint tissues. Therefore, safer and better tolerated agents are needed for OA treatment.

In recent years, interest in OA treatment with plantbased therapy, including traditional Chinese medicine, has been increasing. Generally, a traditional Chinese medicine contains multiple active ingredients that aim at multiple targets [1]. An excellent example of this formula is the $\mathrm{Fu}$
Yuan Capsule (FYC), which is against OA and its related diseases. Our previous studies showed that FYC is an effective drug for knee OA patients [2]. However, the pharmacological mechanism of FYC remains unclear.

A growing body of evidence demonstrates that the extracellular matrix (ECM) degradation factors, inflammatory mediators, and articular cartilage protective factors are all significantly changed in OA. Moreover, a disintegrin and metalloproteinase with thrombospondin motifs (ADAMTS), matrix metalloproteinase (MMP), interleukin-1 beta (IL-1 $\beta$ ), nitric oxide (NO), prostaglandin E2 (PGE2), heat-shock protein 70 (HSP70), transforming growth factor-beta (TGF- $\beta$ ), osteoprotegerin (OPG), and receptor activator of nuclear factor $\kappa$ B ligand (RANKL) play an important role based on their ability to destruct or protect articular cartilage [3-6]. In the present study, the effects of FYC on ECM in a rat OA 
model were investigated. The aforementioned factors possibly involved in the process of OA were specifically assayed.

\section{Materials and Methods}

2.1. Animals and Drug Administration. Forty-five SD rats were obtained from the Animal Center of Chongqing Medical University (Chongqing, China) (certificate number SCXK [Yu] 2007-0001). FYC was mainly composed of Epimedium, Ginseng, Radix Astragali, Pilose antler, Medlar, and Radix Notoginseng. It was produced by Xi Er An Pharmaceutical Company (Chongqing, China). Each capsule weighed $0.41 \mathrm{~g}$, which is equivalent to $3 \mathrm{~g}$ of crude drug. The research preparation approval number for this drug is [2005] $12 \mathrm{~B}$ 55. With the approval of the Institutional Animal Care and Use Committee of Chongqing Medical University, thirty rats underwent anterior cruciate ligament transection in the right posterior knee joint to generate an experimental model of OA as previously described [7]. These rats were then randomly divided into two groups. After two weeks, the rats received daily gavage of saline with or without FYC $(0.3 \mathrm{~mL}$, $2.26 \mathrm{~g} / \mathrm{kg} \cdot \mathrm{d}$ ) for twelve weeks. The remaining fifteen rats were used as controls.

2.2. Histologic Evaluations. Femoral epicondyles were removed, fixed in $4 \%$ paraformaldehyde, then decalcified, and processed through a series of increasing ethanol concentrations for dehydration. These samples were subsequently paraffin-embedded and sectioned at $4 \mu \mathrm{m}$. The sections were then stained with hematoxylin and eosin for general histological assessment, where the grading of hematoxylin and eosin staining was determined according to Mankin's scoring system [8], or with Masson staining for assessment of cartilage collagen and toluidine blue for assessment of cartilage proteoglycan. For the immunohistochemical analysis of anti-rat primary polyclonal antibodies, slides were incubated with types I and II collagen antibodies (Boster Biological Engineering Co., Ltd., Wuhan, China) overnight at $4^{\circ} \mathrm{C}$ in a wet room and then with the secondary antibody (Boster Biological Engineering Co., Ltd., Wuhan, China). The expression of types I and II collagen was visualized by chromogen 3,3-diaminobenzidine immunolabeling. Finally, the sections were counterstained with hematoxylin. The contents of collagen and proteoglycan were quantitatively analyzed with Image-Pro Plus 7.0.

2.3. Sample Hydrolysis Method, Nitrate Reductase Method, and Enzyme-Linked Immunosorbent Assay (ELISA). Hydroxyproline and C-terminal crosslinking telopeptides of type II collagen (CTX-II) are markers of collagen synthesis and degradation. In this study, these markers were measured using sample hydrolysis method kits (Jiancheng Biological Engineering Research Institute, Nanjing, China) and ELISA kits (Hua Mei Biotechnology, Wuhan, China) according to the manufacturer's instructions. ELISA kit for IL-1 $\beta$, PGE2, and $\mathrm{NO}$ assay kit (nitrate reductase method) were purchased from Shengbo Technology Co., Ltd. (Chongqing, China) and
TABLE 1: RT-PCR primers.

\begin{tabular}{|c|c|}
\hline Gene & Sequence \\
\hline \multicolumn{2}{|l|}{ ADAMTS- 4} \\
\hline Sense & 5'GCCTTTCTCTGGTTTGGAGC3' \\
\hline Antisense & $5^{\prime}$ GGCTGGTAATCGGTACAGCA3' \\
\hline \multicolumn{2}{|l|}{ ADAMTS-5 } \\
\hline Sense & 5'TTCCGTCATTAGCCACAGCAG3' \\
\hline Antisense & 5'GTGTCACAGGTCCTAGAGCAG3' \\
\hline \multicolumn{2}{|l|}{ MMP-1 } \\
\hline Sense & 5'ACAGTCCATGGATCCAGGTTATC3' \\
\hline Antisense & 5'CGGAGGCTAAATCTGCGTTG3' \\
\hline \multicolumn{2}{|l|}{ MMP-2 } \\
\hline Sense & 5'GCCCCCATGAAGCCTTGTTT3' \\
\hline Antisense & 5'GCTGGTGCAGCTCTCATACT3' \\
\hline \multicolumn{2}{|l|}{ MMP-3 } \\
\hline Sense & 5'TGTGGTTGTGTGCTCATCCT3' \\
\hline Antisense & 5'CCTGTCATCTCCAACCCGAG3' \\
\hline \multicolumn{2}{|l|}{ MMP-9 } \\
\hline Sense & $5^{\prime}$ GAAAACCTCCAACCTCACGGA3' \\
\hline Antisense & 5'TTTGGAATCGACCCACGTCT3' \\
\hline \multicolumn{2}{|l|}{ MMP-13 } \\
\hline Sense & 5'TGAACATCCATCCCGTGACC $3^{\prime}$ \\
\hline Antisense & 5'ACTCCACACGTGGTTCTCAG3' \\
\hline \multicolumn{2}{|l|}{ IL-1 $\beta$} \\
\hline Sense & 5'ATGCCACCTTTTGACAGTGATG3' \\
\hline Antisense & 5'AGCTTCTCCACAGCCACAAT3' \\
\hline \multicolumn{2}{|l|}{ OPG } \\
\hline Sense & 5'GCCACGCAAAAGTGTGGAAT3' \\
\hline Antisense & 5'TTTGGTCCCAGGCAAACTGT3' \\
\hline \multicolumn{2}{|l|}{ RANKL } \\
\hline Sense & 5'TCGGGAAGCGTACCTACAGA3' \\
\hline Antisense & 5'CCCCAAAGTACGTCGCATCT3' \\
\hline \multicolumn{2}{|l|}{ TGF- $\beta 1$} \\
\hline Sense & 5'AGCTGCGCTTGCAGAGATTA3' \\
\hline Antisense & 5' AGCCCTGTATTCCGTCTCCT3' \\
\hline \multicolumn{2}{|l|}{ GAPDH } \\
\hline Sense & 5'TCAGGAGAGTGTTTCCTCGT3' \\
\hline Antisense & 5'TGCCGTGAGTGGAGTCATAC3' \\
\hline
\end{tabular}

Jiancheng Biological Engineering Research Institute (Nanjing, China), respectively.

2.4. Quantitative Real-Time Polymerase Chain Reaction. Total RNA was extracted using TRIzol reagent (Invitrogen, Shanghai, China) according to the manufacturer's instructions. The reverse transcriptase cDNA synthesis kit (TOYOBO, Japan) was used to obtain cDNA. The $2^{-\Delta \Delta C T}$ method was performed to calculate the relative fold changes in mRNA expression. The sequences of the sense and antisense primers used for amplification are listed in Table 1. 


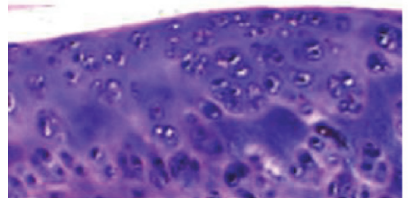

Control

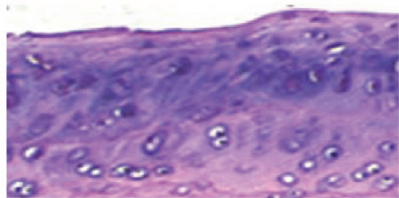

Model

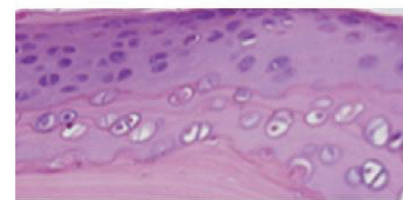

Fu Yuan Capsule

(a)

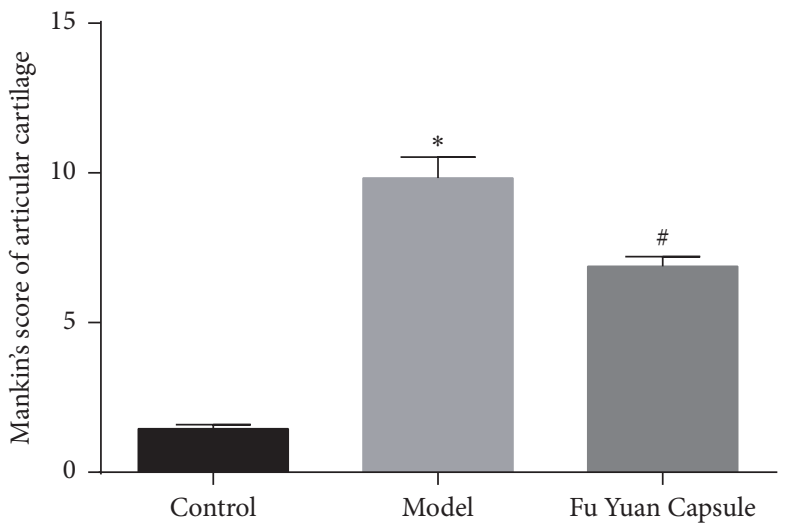

(b)

FIGURE 1: Histological analysis of articular cartilage sections. (a) Hematoxylin-eosin staining of knee joint sections in the control group showed a smooth surface and a clear laminar structure in the articular cartilage. By contrast, the cartilage surface in the model group was rough with some superficial leakage and ulcers. Furthermore, Fu Yuan Capsule-treated cartilage showed fewer lesions compared with the model group (magnification, 200x). (b) Mankin's score of articular cartilage. Data are expressed as mean \pm SD. ${ }^{*} p<0.05$ compared with the control group; ${ }^{*} p<0.05$ compared with the other groups.

2.5. Western Blot Analysis. Proteins were isolated using an extraction kit (Beyotime Institute of Biotechnology, Jiangsu, China). The protein extracts were resolved by sodium dodecyl sulfate-polyacrylamide gel electrophoresis, transferred onto PVDF membranes, and probed with primary antibodies overnight at $4^{\circ} \mathrm{C}$. MMP-1, MMP-3, MMP-13, and $\beta$-actin antibodies were purchased from Proteintech Group (Man, UK). MMP-2, MMP-9, ADAMTS-4, and ADAMTS-5 antibodies were obtained from Santa Cruz (USA). HSP70 antibody was purchased from Boster Biological Engineering Co., Ltd. (Wuhan, China). The membranes were washed with TBS containing $0.05 \%$ Tween 20 and incubated with the appropriate HPR-linked secondary antibodies at $37^{\circ} \mathrm{C}$ for $1 \mathrm{~h}$, followed by visualization using an enhanced chemiluminescence kit (Beyotime Institute of Biotechnology, Jiangsu, China).

2.6. Statistical Analysis. Data are expressed as mean \pm standard deviation. Comparisons were made using one-way analysis of variance with SPSS version 18.0 for Windows (SPSS Inc., Chicago, IL, USA). A $p$ value less than 0.05 was considered significant.

\section{Results}

3.1. Effects of FYC on Joint Structure. The effect of FYC on the rat $\mathrm{OA}$ model was confirmed by histological analyses. Figure 1(a) reveals that the model group exhibited some structural changes (superficial leakage and ulcers) in the articular cartilage compared with the control group. Moreover, treatment with FYC was associated with less damage in the cartilage compared with the model group. Figure 1(b) shows the histological scores associated with the histological studies.

3.2. Effects of FYC on ECM. Types I (Figure 2(a)) and II collagen (Figure 2(b)), collagen (Figure 2(c)), proteoglycan (Figure 2(c)), CTX-II, and hydroxyproline (Figure 3) were determined to identify the effect of FYC on ECM in the rat OA model. The results showed that the model group had significantly higher levels of type I collagen and CTX-II but lower levels of type II collagen, collagen, proteoglycan, and hydroxyproline as compared with the control group. Compared with the model group, the FYC group showed significantly decreased type I collagen and CTX-II levels and increased type II collagen, collagen, proteoglycan, and hydroxyproline levels.

3.3. Effect of FYC on ECM Degradation Factors. To determine ECM degradation factors under different conditions, we measured the expression of ADAMTS-4, ADAMTS-5, MMP-1, MMP-2, MMP-3, MMP-9, and MMP-13 in OA cartilage (Figures 4 and 5). Compared with the control group, the model 


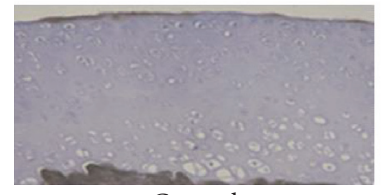

Control

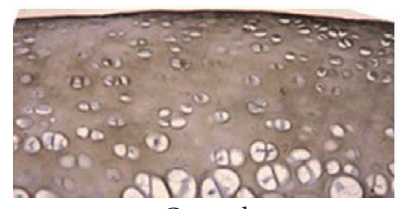

Control

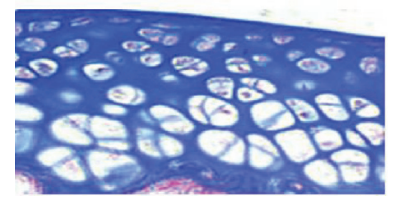

Control

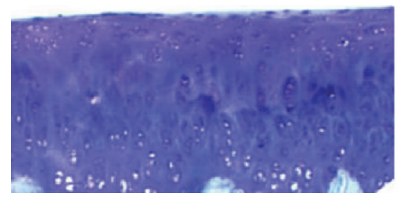

Control

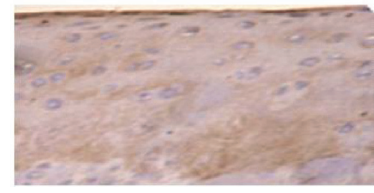

Model

(a)

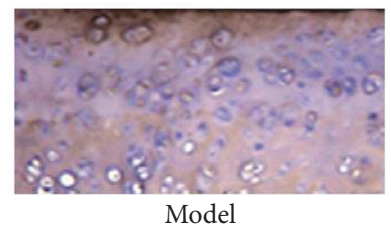

(b)

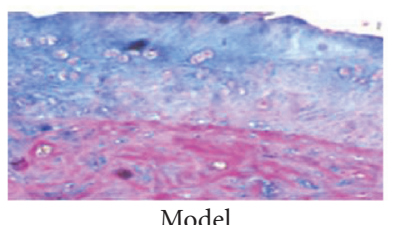

(c)

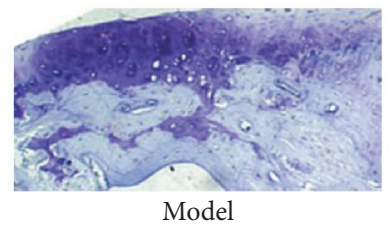

(d)

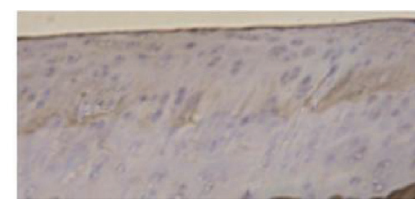

Fu Yuan Capsule

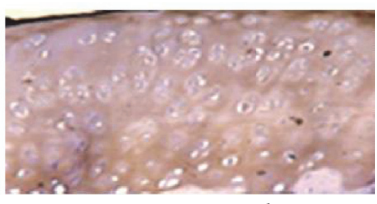

Fu Yuan Capsule

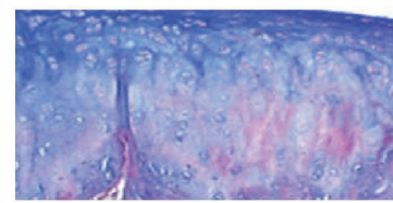

Fu Yuan Capsule

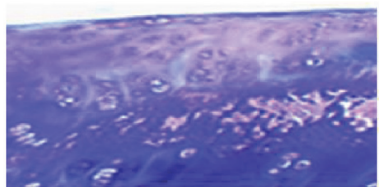

Fu Yuan Capsule

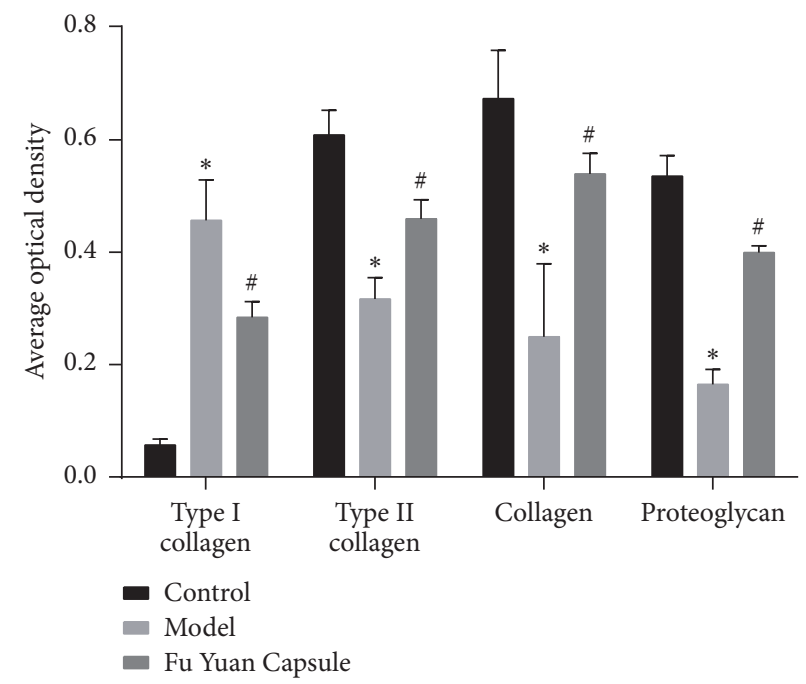

(e)

Figure 2: Effects of Fu Yuan Capsule on extracellular matrix in a rat osteoarthritis model. (a) Immunohistochemical staining of type I collagen (magnification, 200x). (b) Immunohistochemical staining of type II collagen (magnification, 200x). (c) Masson staining of collagen (magnification, 200x). (d) Toluidine blue staining of proteoglycan (magnification, 200x). (e) Average optical density of staining was calculated using image analysis software Image-Pro Plus 7.0. Data are expressed as mean \pm SD. ${ }^{*} p<0.05$ compared with the control group; ${ }^{\#} p<0.05$ compared with the other groups. 


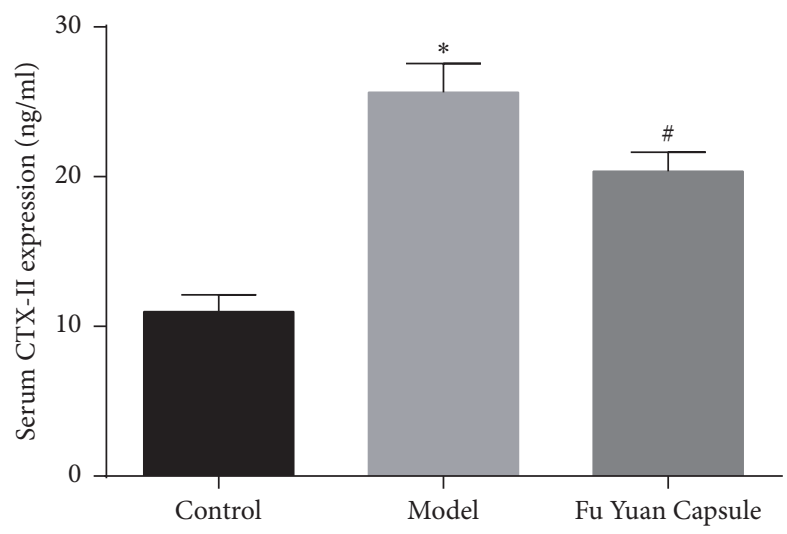

(a)

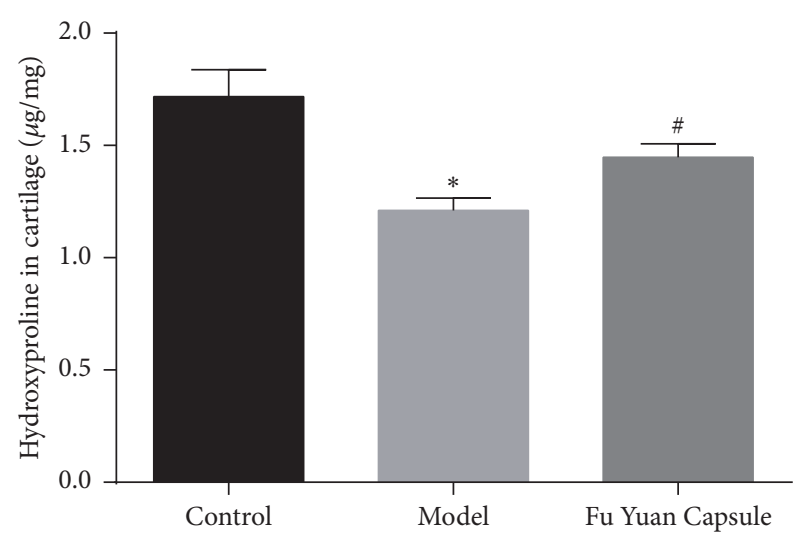

(b)

FIGURE 3: Effects of Fu Yuan Capsule on CTX-II and hydroxyproline in a rat osteoarthritis model. (a) ELISA detected CTX-II in serum. (b) Hydroxyproline was measured by a sample hydrolysis method. Data are expressed as mean $\pm \mathrm{SD}$ (each group, $n=3$ ). ${ }^{*} p<0.05$ compared with the control group; ${ }^{\#} p<0.05$ compared with the other groups.

group showed significantly increased ECM degradation factors. The increased factors in the model group were restrained by FYC.

3.4. Effect of FYC on Inflammatory Mediators. IL-1 $\beta, \mathrm{NO}$, and PGE2 are important inflammatory mediators in OA. They were significantly increased in the model group as compared with those in the control group. Likewise, increased inflammatory mediators were reversed by FYC (Figure 6).

\subsection{Effect of FYC on Articular Cartilage Protective Factor.} TGF- $\beta$, HSP70, and OPG play an important role in articular cartilage protection. As shown in Figure 7, the extent of TGF$\beta$, HSP70, and OPG/RANKL ratio was significantly increased as compared with that in the model and control groups.

\section{Discussion}

$\mathrm{OA}$ is a heterogeneous, complicated joint disorder whose pathogenesis is affected by multiple etiologies, including aging and obesity, as well as by mechanical, biochemical, and genetic factors [9]. Traditional Chinese medicine has fought against $\mathrm{OA}$ and its related diseases for many years in China. Our research group has been focusing on the role of FYC in OA. In our previous clinical research, the effectiveness and safety of FYC for OA were identified based on an observational clinical research [2]. In the present study, we analyzed the effect of FYC on collagen and proteoglycan, including ADAMTS, MMPs, IL-1 $\beta$, NO, PGE2, TGF- $\beta$, HSP70, OPG, and RANKL levels, which are involved in ECM degradation, as well as articular cartilage apoptotic and articular cartilage protective pathways. The experimental results showed that FYC could effectively protect ECM, reduce ECM degradation factors, depress inflammatory mediators, and increase articular cartilage protective factor in a rat OA model.

Articular cartilage degradation is the most predominant change observed in OA. To our knowledge, this study is the first to use a variety of methods to detect the effect of
FYC on collagen and proteoglycan. Proteoglycans and type II collagen are the major constituents of cartilage. Type II collagen is localized almost exclusively in cartilage. Thus, measurements of fragments derived from this protein may potentially represent a specific marker for cartilage degradation. Previous studies reported that the concentrations of CTX-II in urine and serum reveal the extent of cartilage damage and are a good marker of OA progression [10-13]. The FYC could protect the collagen and proteoglycan of the articular cartilage in a rat OA model in the current study. This result is consistent with that in the rabbit OA model in our previous study. Epimedium and Ginseng are the major components of FYC. Other studies found that they have a protective effect on articular cartilage in OA [14, 15]. This finding also supports our results from another perspective.

The mechanistic details of OA pathogenesis remain to be elucidated. ECM degradation is an important contributing factor in articular cartilage injury. The ADAMTS family is known to influence the development, angiogenesis, coagulation, and progression of arthritis. Among the 19 members of ADAMTS family, ADAMTS- 4 and ADAMTS- 5 have received significant attention in the pathology of arthritic joint diseases because they are the most efficient aggrecanases in vitro. Several experiments have demonstrated that neutralizing antibodies and other methods play a significant role in the models of arthritis in restraining ADAMTS- 4 or ADAMTS-5 [3]. ADAMTS are the main proteinases responsible for aggrecan cleavage in the early events of cartilage remodeling. The MMPs start participating in this process during the development of the disease and continue with collagen degradation. MMP-1, MMP-3, and MMP-13 are more restricted to cartilage and drive OA progression; they not only target the collagens in cartilage for degradation, but also degrade proteoglycan, osteonectin, and perlecan in cartilage [16]. In previous decades, numerous MMP inhibitors have been suggested as candidates for OA treatment. The results in this study are consistent with a role for these factors in regulating articular cartilage in OA. Moreover, the present results demonstrated that the protective effect of FYC on ECM was 


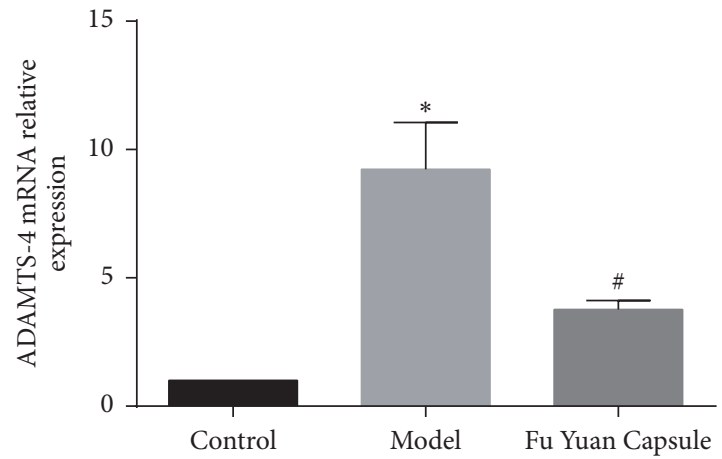

(a)

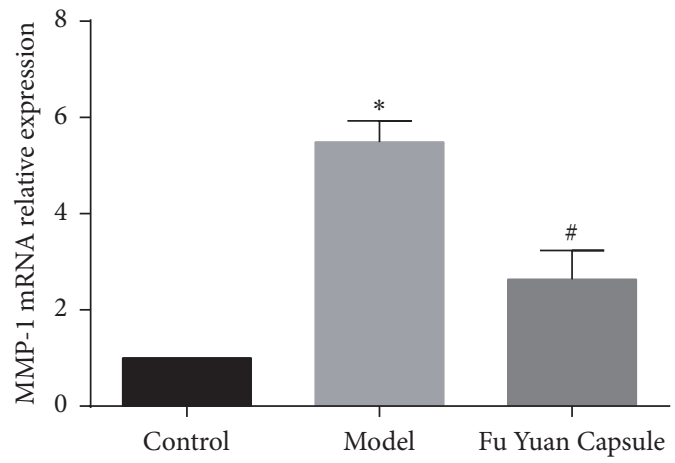

(c)

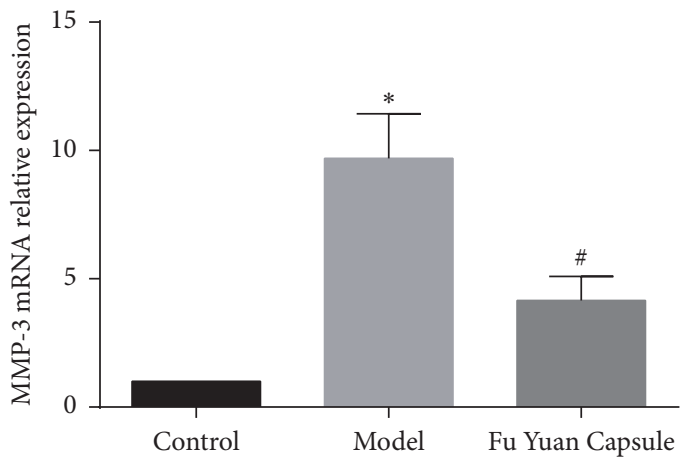

(e)

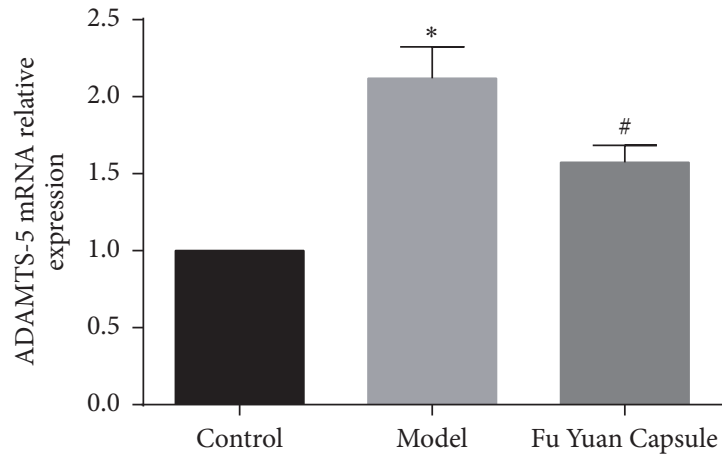

(b)

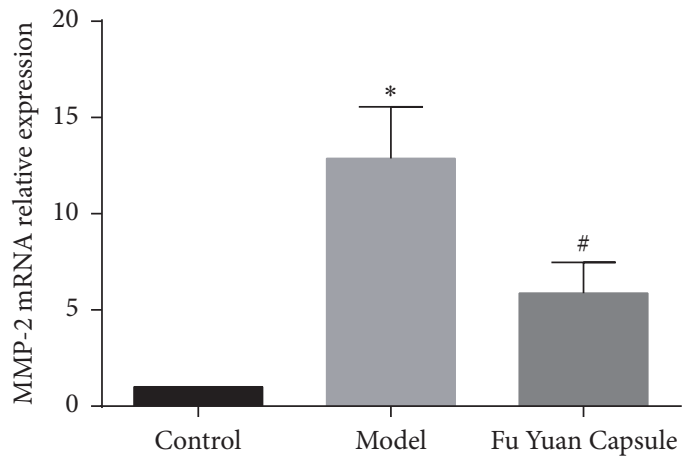

(d)

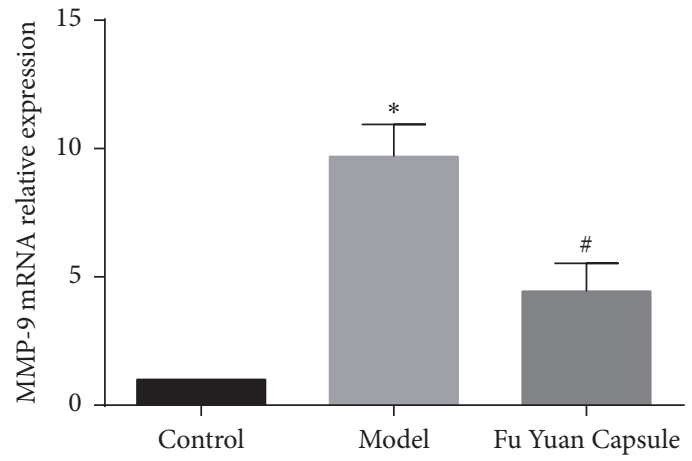

(f)

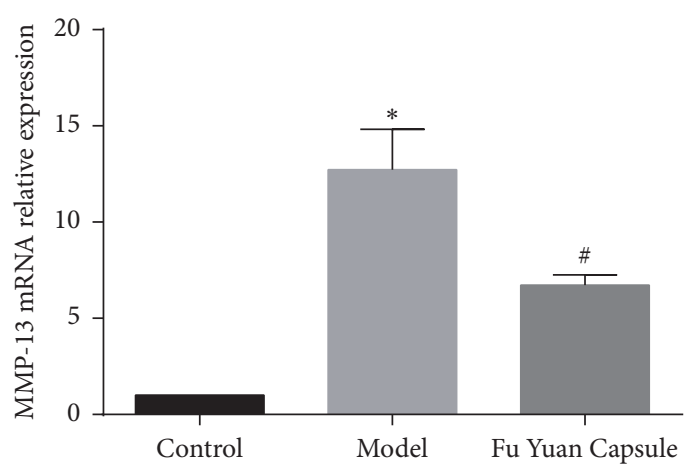

(g)

Figure 4: Effects of Fu Yuan Capsule on mRNA expression of extracellular matrix degradation factors. (a), (b), (c), (d), (e), (f), and (g), respectively, showed that ADAMTS-4, ADAMTS-5, MMP-1, MMP-2, MMP-3, MMP-9, and MMP-13 mRNA were detected by quantitative real-time polymerase chain reaction. Data are expressed as mean $\pm \mathrm{SD}$ (each group, $n=3$ ). ${ }^{*} p<0.05$ compared with the control group; ${ }^{\#} p<0.05$ compared with the other groups. 


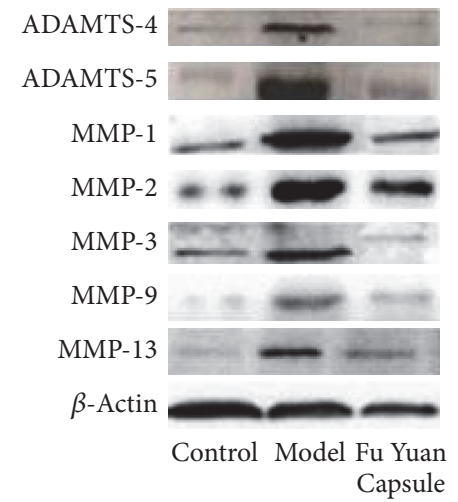

(a)

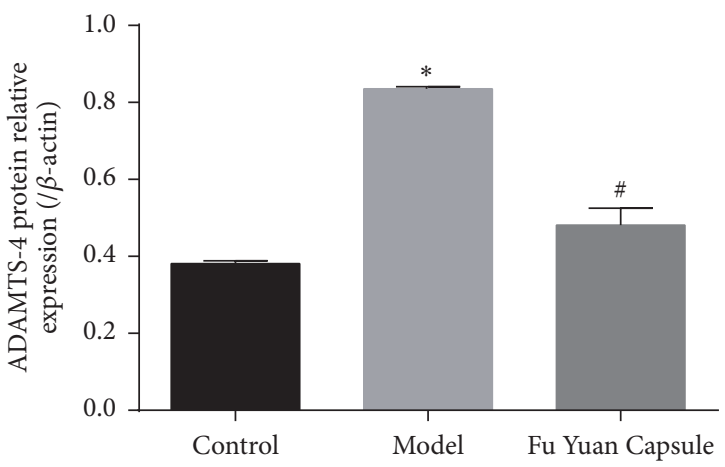

(b)

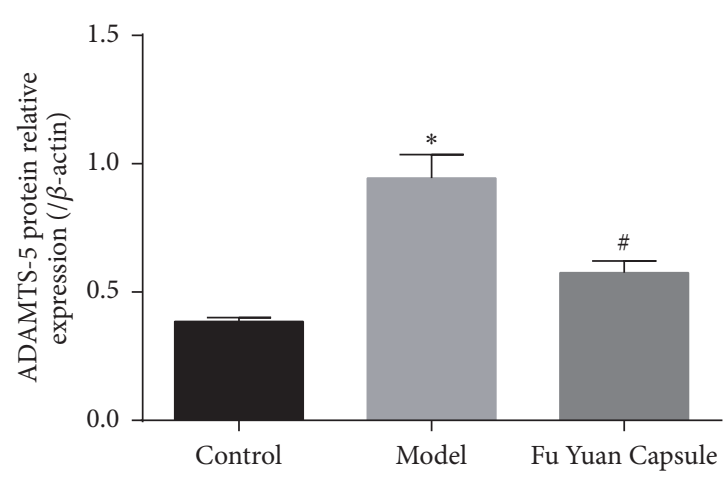

(c)

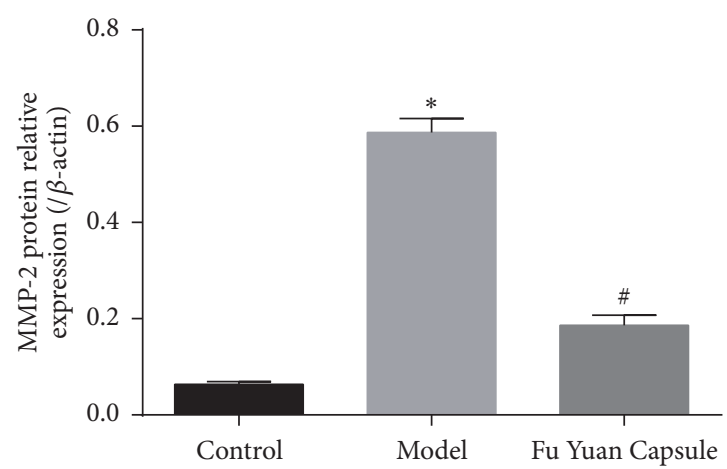

(e)

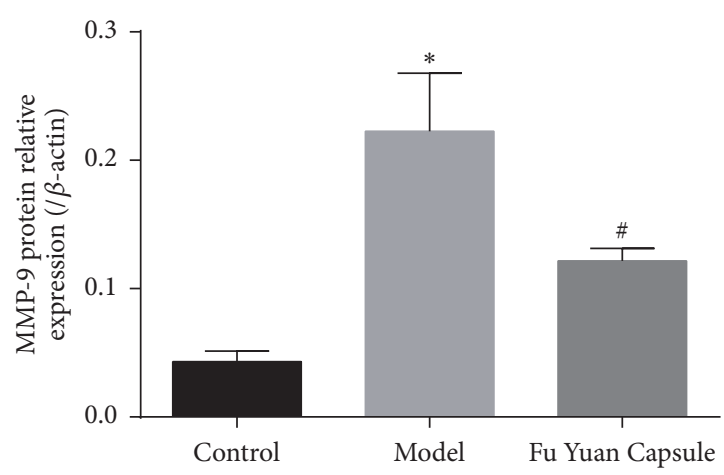

(g)

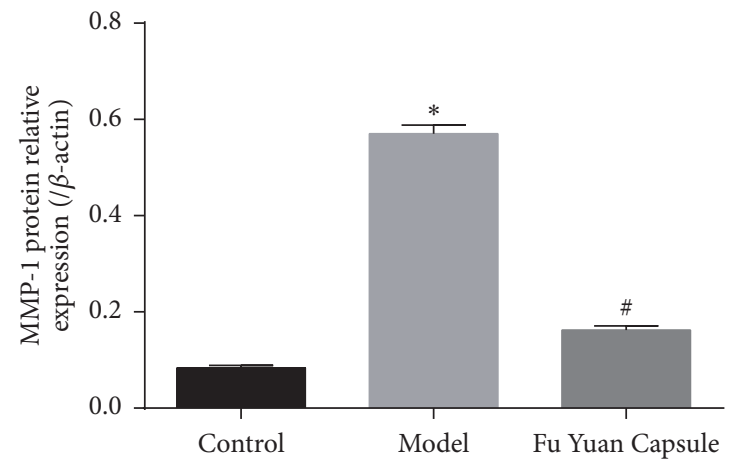

(d)

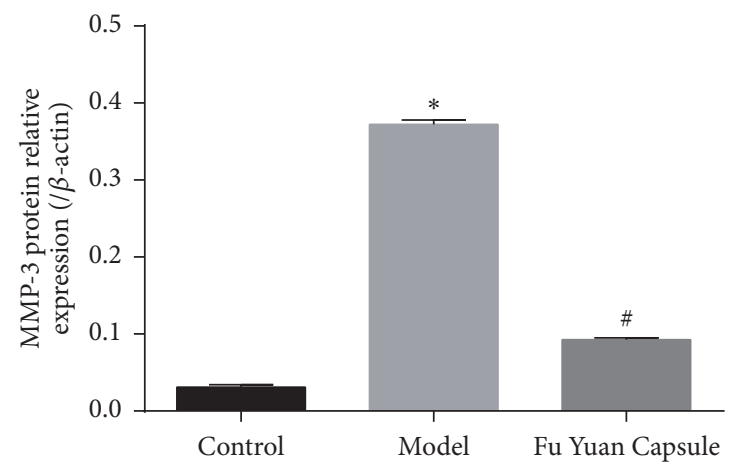

(f)

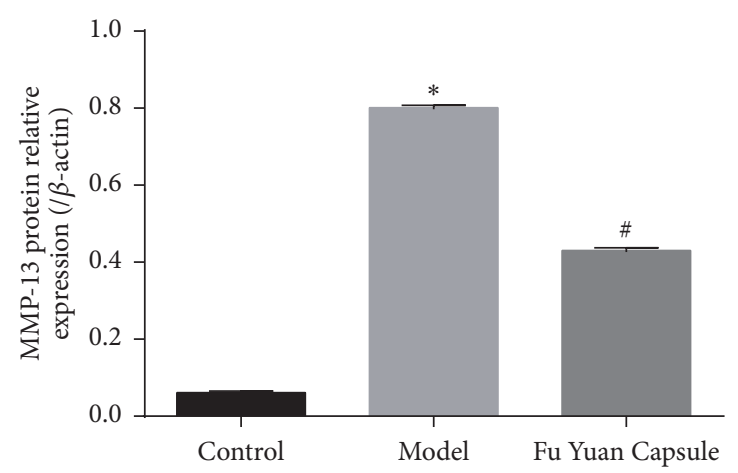

(h)

Figure 5: Effects of Fu Yuan Capsule on protein expression of extracellular matrix degradation factors. Representative Western blotting bands (a) and semiquantitative analysis of ADAMTS-4 (b), ADAMTS-5 (c), MMP-1 (d), MMP-2 (e), MMP-3 (f), MMP-9 (g), and MMP-13 (h) in various groups. (a) Each row represents images cropped from different gels probed with different antibodies. Data are expressed as mean \pm $\mathrm{SD}$ (each group, $n=3$ ). ${ }^{*} p<0.05$ compared with the control group; ${ }^{*} p<0.05$ compared with the other groups. 


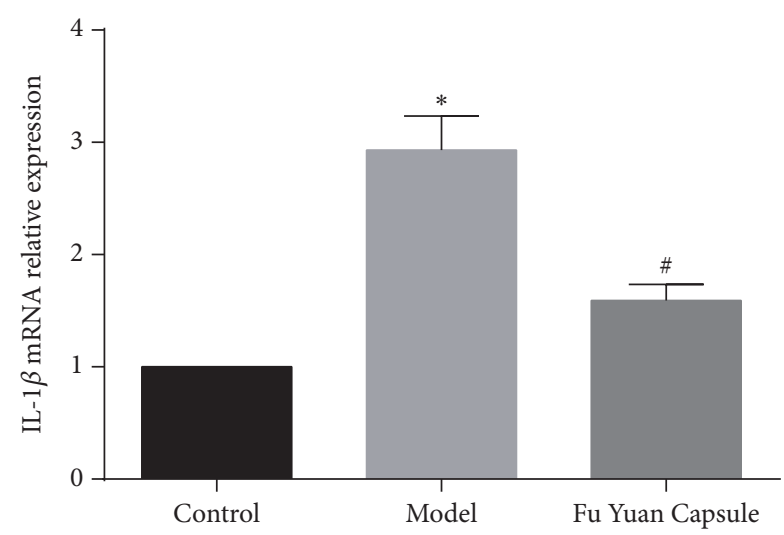

(a)

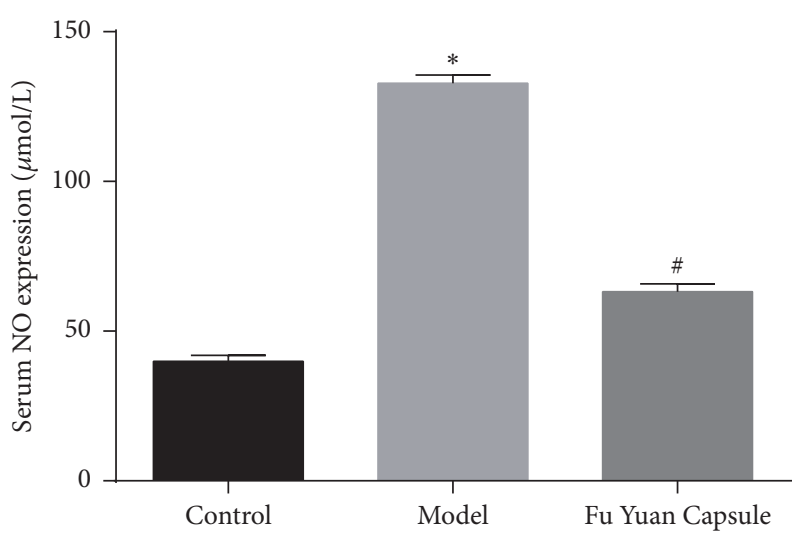

(c)

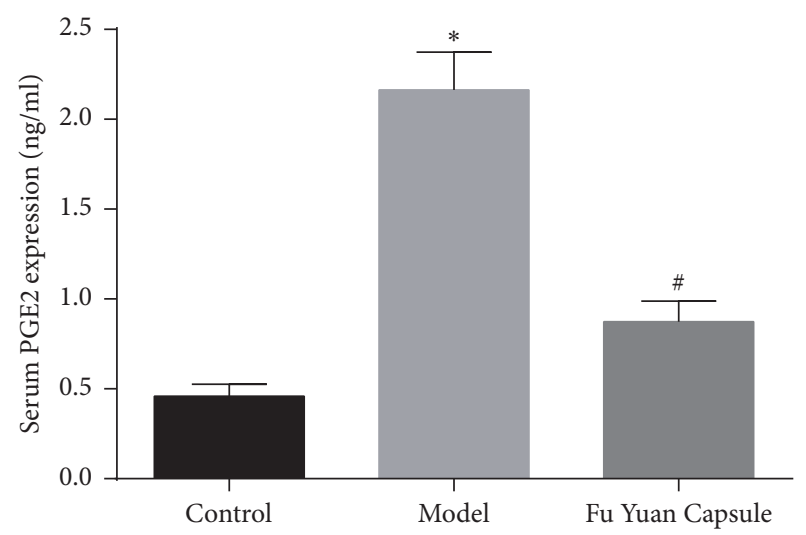

(e)

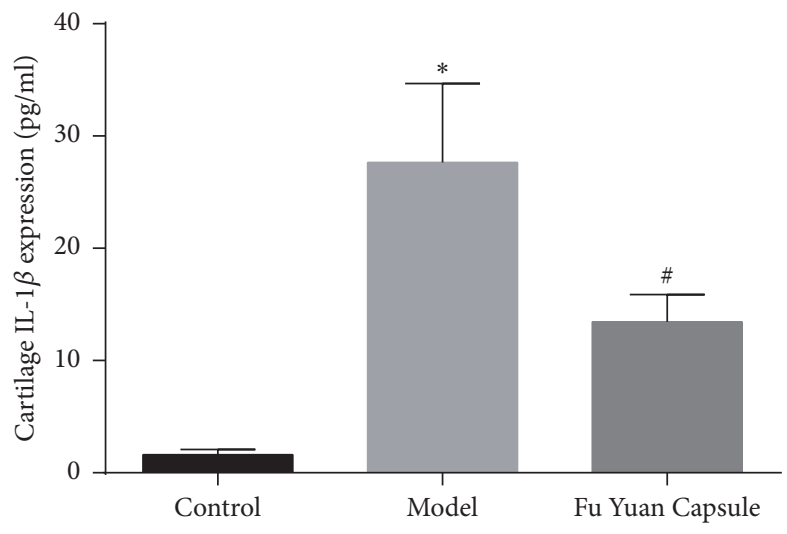

(b)

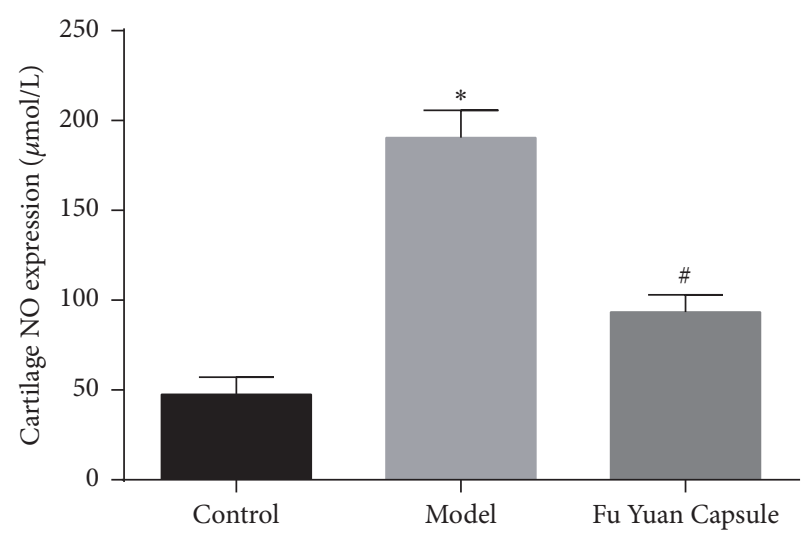

(d)

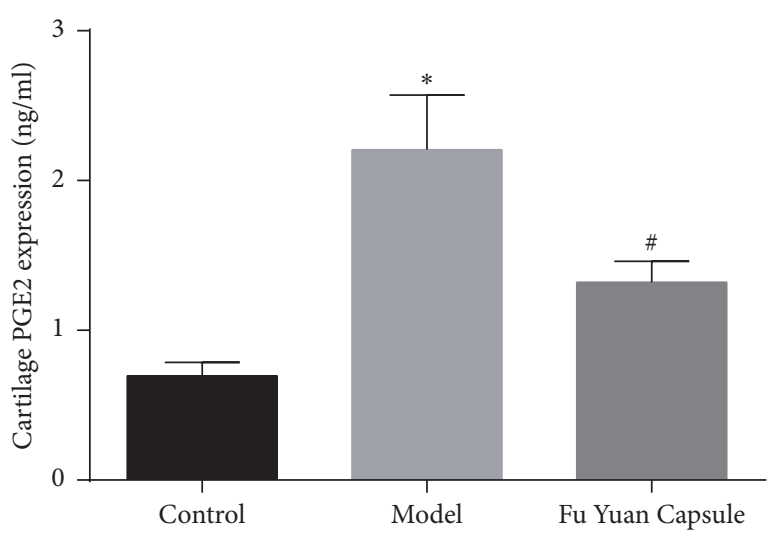

(f)

FIGURE 6: Effects of Fu Yuan Capsule on inflammatory mediators. IL-1 $\beta$ mRNA (a) was detected by quantitative real-time polymerase chain reaction. The expression of NO (c) and PGE2 (e) was detected by enzyme-linked immunosorbent assay in serum. The expression of IL-1 $\beta$ (b), NO (d), and PGE2 (f) was detected by enzyme-linked immunosorbent assay in cartilage. Data are expressed as mean \pm SD (each group, $n=3) .{ }^{*} p<0.05$ compared with the control group; ${ }^{*} p<0.05$ compared with the other groups.

partially achieved by inhibiting ADAMTS-4, ADAMTS-5, MMP-1, MMP-2, MMP-3, MMP-9, and MMP-13.

The past decade has seen a gradual shift in our understanding of the mechanisms underlying OA. We now know that this disease affects the entire joint structure, and inflammation has a critical role in its pathogenesis [17]. IL- $1 \beta$ is a pleiotropic proinflammatory cytokine that plays a significant role in the development of OA by stimulating several mediators that contribute to cartilage degradation. NO and PGE2 are important inflammatory mediators in OA pathogenesis. Overproductions of $\mathrm{NO}$ and PGE2 are correlated to the pathophysiology of OA. IL- $1 \beta$ could significantly increase the production of $\mathrm{NO}$ and PGE2. Some potential agents in OA treatment could effectively inhibit the IL- $1 \beta$-induced 


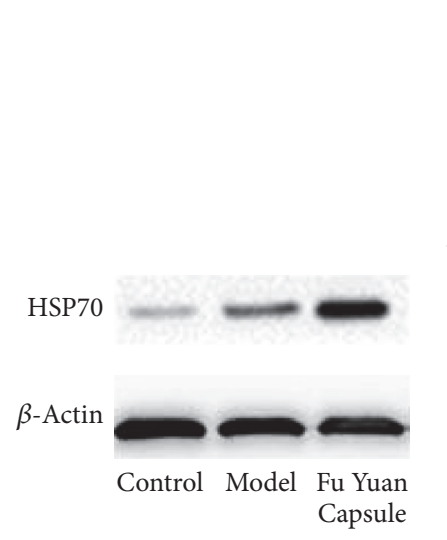

(a)

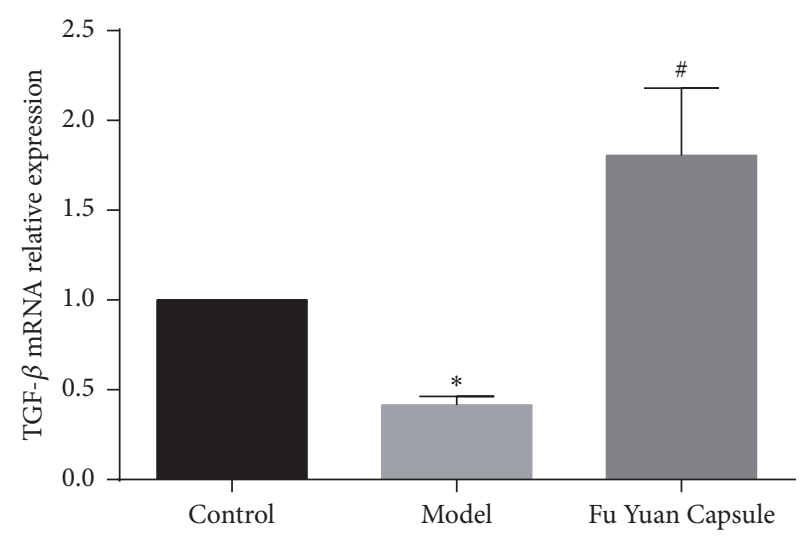

(c)

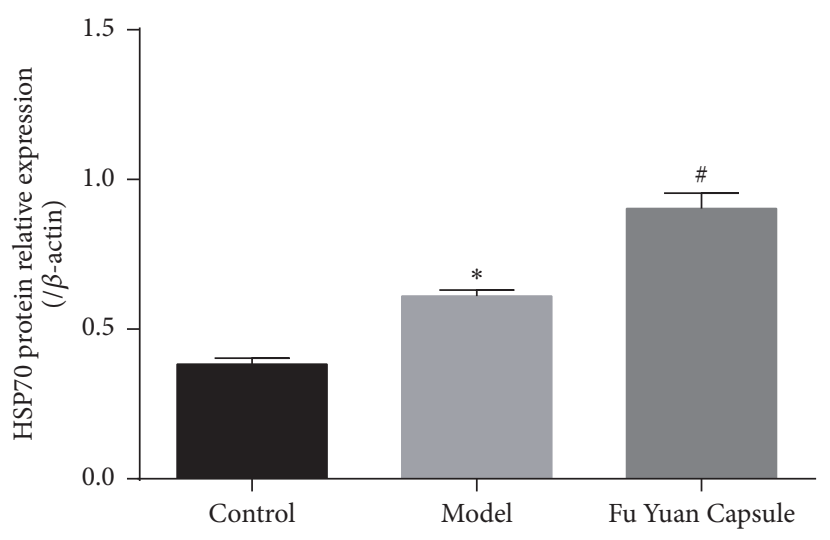

(b)

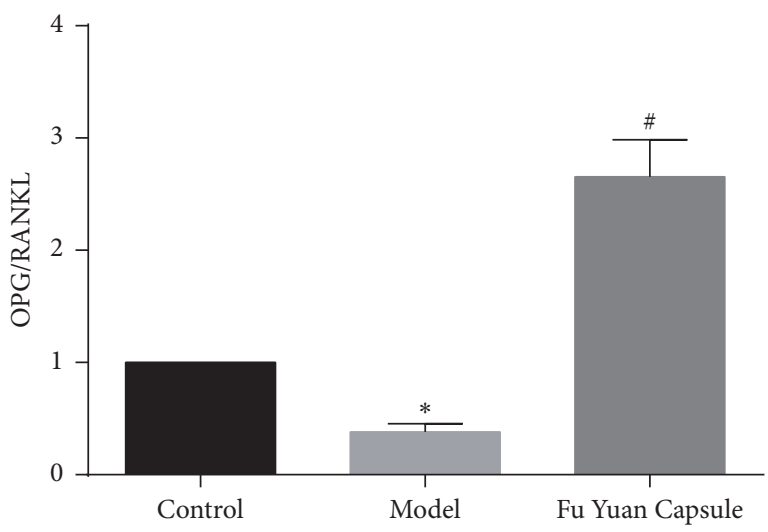

(d)

FiguRE 7: Effects of Fu Yuan Capsule on articular cartilage protective factor. Representative Western blotting bands (a) and semiquantitative analysis of HSP70 (b) in various groups. TGF- $\beta$ mRNA (c) was detected by quantitative real-time polymerase chain reaction. Quantitative realtime polymerase chain reaction analysis was also performed to examine the mRNA expression of OPG and RANKL (d). Data are expressed as mean $\pm \mathrm{SD}$ (each group, $n=3$ ). ${ }^{*} p<0.05$ compared with the control group; ${ }^{*} p<0.05$ compared with the other groups.

expression of inflammatory mediators, including $\mathrm{NO}$ and PGE2 [18]. Several studies found that the expression of IL$1 \beta, N O$, and PGE2 is significantly increased in arthritis tissue and OA animal models, even in patient serum [12, 19-22]. In addition to the local inflammatory mediators through the bloodstream, the essence of inflammation in OA involves the interplay of the innate immune system and inflammatory mediators, which cause chronic, comparatively low-grade, systemic inflammation [22]. This may be the most important reason for the increase in inflammatory mediators in serum. Combined with the results of this study, we found that FYC possesses anti-inflammatory effect in OA.

Articular cartilage also has some protection mechanisms in OA. TGF- $\beta$ is a multifunctional cytokine involved in crucial biological processes, such as ECM synthesis, cell proliferation and differentiation, and tissue repair $[23,24]$. Intraarticular injection of TGF- $\beta$ increases proteoglycan synthesis and articular cartilage content in murine knee joints. Loss of TGF- $\beta$ signaling in cartilage induces chondrocyte hypertrophy, which leads to cartilage degeneration, and activation of the TGF- $\beta$ pathway has therefore been proposed to preserve articular cartilage integrity during OA [4]. HSP70 has been reported to be highly expressed in OA chondrocytes. It has the potential to prevent cartilage damage in arthritic joints and inhibit nitric oxide-induced apoptosis in chondrocytes by reducing caspase 3 activity [5]. Other studies further found that HSP70 promotes the expression of type II collagen and proteoglycan core protein in chondrocytes. RANKL, a member of the tumor necrosis factor family, can activate nuclear factor- $\kappa \beta$ ligand through RANK-RANKL specific binding to stimulate osteoclast activation. OPG, a membrane-bound tumor necrosis factor-related factor, is an important cytokine that protects the cartilage and plays a vital role in regulating the biology of cartilaginous tissues [25]. It works as a decoy receptor for RANKL and therefore prevents RANK activation and subsequent osteoclastogenesis $[6,26]$. The OPG/RANKL ratio is an index of osteoclastogenic stimulus. In this study, high levels of TGF- $\beta$, HSP70 and OPG/RANKL ratio were detected in the FYC group. Therefore, the protective role of FYC on articular cartilage was further confirmed.

\section{Conclusion}

Given that traditional Chinese medicine is composed of multiactive components and possesses multitarget action feature, it can aim at multiple targets, exerting a systemic effect at the 
same time. Multitarget medicine has been proven to be particularly effective in treating complex diseases [1, 27-29]. In this study, we found that FYC protected articular cartilage via numerous ways, including the reduction of ECM degradation factors, decrease in inflammatory mediator depression, and improvement of articular cartilage protective factor. FYC may be an effective agent for OA treatment.

The fact that FYC could protect the articular cartilage from injury by OA requires more studies. The useful compounds are still unknown, and a deeper insight into the deeper mechanism of FYC in OA should be provided. Several studies have indicated that nuclear magnetic resonancebased metabonomics analysis is an effective approach to assess the therapeutic effects of traditional Chinese medicine [30]. We look forward to do this work in the near future.

\section{Competing Interests}

The authors declare that there is no conflict of interests regarding the publication of this paper.

\section{Acknowledgments}

The authors would like to thank Professors Wei-Xue Tang and Li-Xue Chen for their technical assistance.

\section{References}

[1] J. R. Chen, J. Wei, L. Y. Wang et al., "Cardioprotection against ischemia/reperfusion injury by QiShenYiQi Pill ${ }^{\circledR}$ via ameliorate of multiple mitochondrial dysfunctions," Drug Design, Development and Therapy, vol. 9, pp. 3051-3066, 2015.

[2] L. Zeng and R. H. Li, "Clinical study of Fuyuan capsule on the treatment of knee osteoarthritis," Journal of Practical Traditional Chinese Medicine, vol. 30, no. 8, pp. 692-693, 2014.

[3] P. Verma and K. Dalal, "ADAMTS-4 and ADAMTS-5: key enzymes in osteoarthritis," Journal of Cellular Biochemistry, vol. 112, no. 12, pp. 3507-3514, 2011.

[4] M. Kapoor, J. Martel-Pelletier, D. Lajeunesse, J.-P. Pelletier, and H. Fahmi, "Role of proinflammatory cytokines in the pathophysiology of osteoarthritis," Nature Reviews Rheumatology, vol. 7, no. 1, pp. 33-42, 2011.

[5] N. Hiraoka, Y. Arai, K. A. Takahashi et al., "Mild electrical stimulation with heat stimulation increase heat shock protein 70 in articular chondrocyte," Journal of Orthopaedic Research, vol. 31, no. 6, pp. 894-900, 2013.

[6] P. M. van der Kraan and W. B. van den Berg, "Chondrocyte hypertrophy and osteoarthritis: role in initiation and progression of cartilage degeneration?" Osteoarthritis and Cartilage, vol. 20, no. 3, pp. 223-232, 2012.

[7] H. Jo, H. J. Ahn, E. M. Kim et al., "Effects of dehydroepiandrosterone on articular cartilage during the development of osteoarthritis," Arthritis \& Rheumatism, vol. 50, no. 8, pp. 25312538, 2004.

[8] H. J. Mankin, H. Dorfman, L. Lippiello, and A. Zarins, "Biochemical and metabolic abnormalities in articular cartilage from osteo-arthritic human hips. II. Correlation of morphology with biochemical and metabolic data," The Journal of Bone \& Joint Surgery-American Volume, vol. 53, no. 3, pp. 523-537, 1971.
[9] P. M. van der Kraan, "Understanding developmental mechanisms in the context of osteoarthritis," Current Rheumatology Reports, vol. 15, no. 6, article 333, 2013.

[10] S. Oestergaard, B. C. Sondergaard, P. Hoegh-Andersen et al., "Effects of ovariectomy and estrogen therapy on type II collagen degradation and structural integrity of articular cartilage in rats: implications of the time of initiation," Arthritis \& Rheumatism, vol. 54, no. 8, pp. 2441-2451, 2006.

[11] M. E. Duclos, O. Roualdes, R. Cararo, J. C. Rousseau, T. Roger, and D. J. Hartmann, "Significance of the serum CTX-II level in an osteoarthritis animal model: a 5-month longitudinal study," Osteoarthritis and Cartilage, vol. 18, no. 11, pp. 1467-1476, 2010.

[12] M. L. Ferrándiz, M. C. Terencio, M. C. Carceller et al., "Effects of BIS076 in a model of osteoarthritis induced by anterior cruciate ligament transection in ovariectomised rats," $B M C$ Musculoskeletal Disorders, vol. 16, no. 1, article 92, 2015.

[13] Y. M. Park, S. J. Kim, K. J. Lee, S. S. Yang, B.-H. Min, and H. C. Yoon, "Detection of CTX-II in serum and urine to diagnose osteoarthritis by using a fluoro-microbeads guiding chip," Biosensors and Bioelectronics, vol. 67, pp. 192-199, 2015.

[14] J. H. Lee, H. Lim, O. Shehzad, Y. S. Kim, and H. P. Kim, "Ginsenosides from Korean red ginseng inhibit matrix metalloproteinase-13 expression in articular chondrocytes and prevent cartilage degradation," European Journal of Pharmacology, vol. 724, no. 1, pp. 145-151, 2014.

[15] L. Zeng, W. Wang, X.-F. Rong et al., "Chondroprotective effects and multi-target mechanisms of Icariin in IL-1 beta-induced human SW 1353 chondrosarcoma cells and a rat osteoarthritis model," International Immunopharmacology, vol. 18, no. 1, pp. 175-181, 2014.

[16] C. Amălinei, I.-D. Căruntu, S. E. Giuşcă, and R. A. Bălan, "Matrix metalloproteinases involvement in pathologic conditions," Romanian Journal of Morphology and Embryology, vol. 51, no. 2, pp. 215-228, 2010.

[17] P. Wojdasiewicz, Ł. A. Poniatowski, and D. Szukiewicz, "The role of inflammatory and anti-inflammatory cytokines in the pathogenesis of osteoarthritis," Mediators of Inflammation, vol. 2014, Article ID 561459, 19 pages, 2014.

[18] L. Wang, T. Ma, Y. Zheng, S. Lv, Y. Li, and S. Liu, "Diosgenin inhibits IL-1 $\beta$-induced expression of inflammatory mediators in human osteoarthritis chondrocytes," International Journal of Clinical and Experimental Pathology, vol. 8, no. 5, pp. 48304836, 2015.

[19] X. Tang, Z. Zhou, B. Shen et al., "Serum levels of TNF- $\alpha$, IL$1 \beta$, COMP, and CTX-II in patients with Kashin-Beck disease in Sichuan, China," Rheumatology International, vol. 32, no. 11, pp. 3503-3509, 2012.

[20] J.-K. Kim, S.-W. Park, J.-W. Kang et al., "Effect of GCSB-5, a herbal formulation, on monosodium iodoacetate-induced osteoarthritis in rats," Evidence-based Complementary and Alternative Medicine, vol. 2012, Article ID 730907, 11 pages, 2012.

[21] J. Salvatierra, G. Escames, P. Hernandez et al., "Cartilage and serum levels of nitric oxide in patients with hip osteoarthritis," Journal of Rheumatology, vol. 26, no. 9, pp. 2015-2017, 1999.

[22] W. H. Robinson, C. M. Lepus, Q. Wang et al., "Low-grade inflammation as a key mediator of the pathogenesis of osteoarthritis," Nature Reviews Rheumatology, vol. 12, no. 10, pp. 580-592, 2016.

[23] A. Villalvilla, A. García-Martín, R. Largo, O. Gualillo, G. Herrero-Beaumont, and R. Gómez, "The adipokine lipocalin2 in the context of the osteoarthritic osteochondral junction," Scientific Reports, vol. 6, Article ID 29243, 2016. 
[24] A. R. Sharma, S. Jagga, S.-S. Lee, and J.-S. Nam, "Interplay between cartilage and subchondral bone contributing to pathogenesis of osteoarthritis," International Journal of Molecular Sciences, vol. 14, no. 10, pp. 19805-19830, 2013.

[25] B. Bolon, M. Grisanti, K. Villasenor, S. Morony, U. Feige, and W. S. Simonet, "Generalized degenerative joint disease in osteoprotegerin (Opg) null mutant mice," Veterinary Pathology, vol. 52, no. 5, pp. 873-882, 2015.

[26] C.-C. Yeh, Y.-H. Su, Y.-J. Lin et al., "Evaluation of the protective effects of curcuminoid (Curcumin and bisdemethoxycurcumin)-loaded liposomes against bone turnover in a cellbased model of osteoarthritis," Drug Design, Development and Therapy, vol. 9, pp. 2285-2300, 2015.

[27] H.-L. He, D. Wang, J. Tang, X.-M. Zhou, J.-X. Li, and L. Xu, "Jin Fu Kang oral liquid inhibits lymphatic endothelial cells formation and migration," Evidence-Based Complementary and Alternative Medicine, vol. 2016, Article ID 3635209, 10 pages, 2016.

[28] C. JianXin, X. Xue, L. ZhongFeng et al., "Qishen Yiqi Drop Pill improves cardiac function after myocardial ischemia," Scientific Reports, vol. 6, Article ID 24383, 2016.

[29] R. Ma, R. Zhu, L. Wang et al., "Diabetic osteoporosis: a review of its traditional chinese medicinal use and clinical and preclinical research," Evidence-Based Complementary and Alternative Medicine, vol. 2016, 13 pages, 2016.

[30] X.-F. Zheng, J.-S. Tian, P. Liu, J. Xing, and X.-M. Qin, "Analysis of the restorative effect of Bu-zhong-yi-qi-tang in the spleenqi deficiency rat model using ${ }^{1} \mathrm{H}-\mathrm{NMR}$-based metabonomics," Journal of Ethnopharmacology, vol. 151, no. 2, pp. 912-920, 2014. 


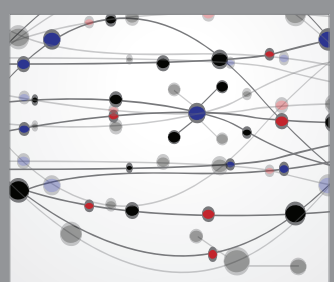

The Scientific World Journal
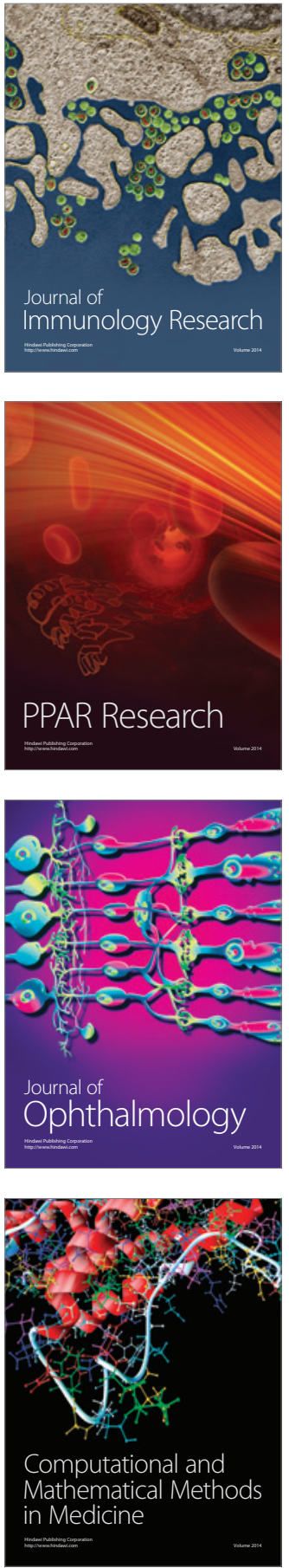

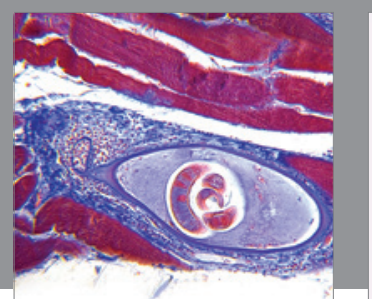

Gastroenterology Research and Practice
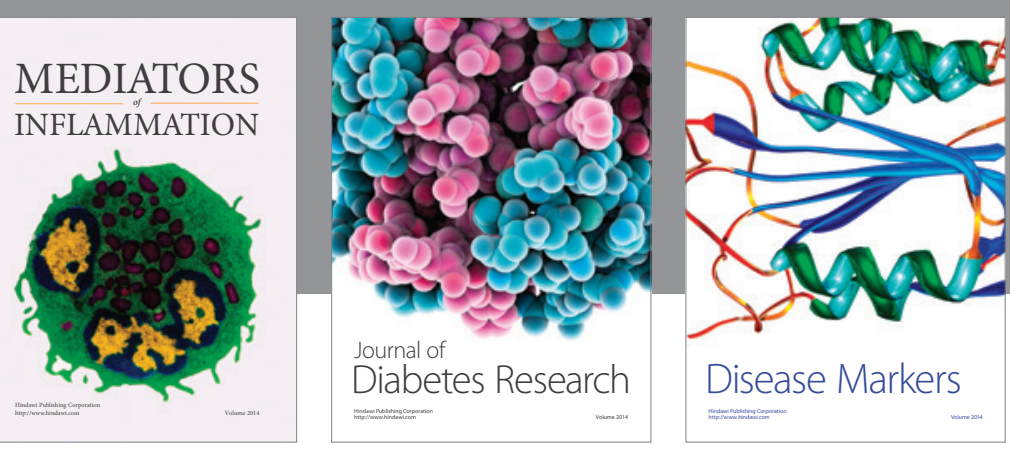

Disease Markers

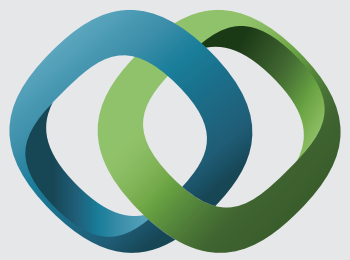

\section{Hindawi}

Submit your manuscripts at

https://www.hindawi.com
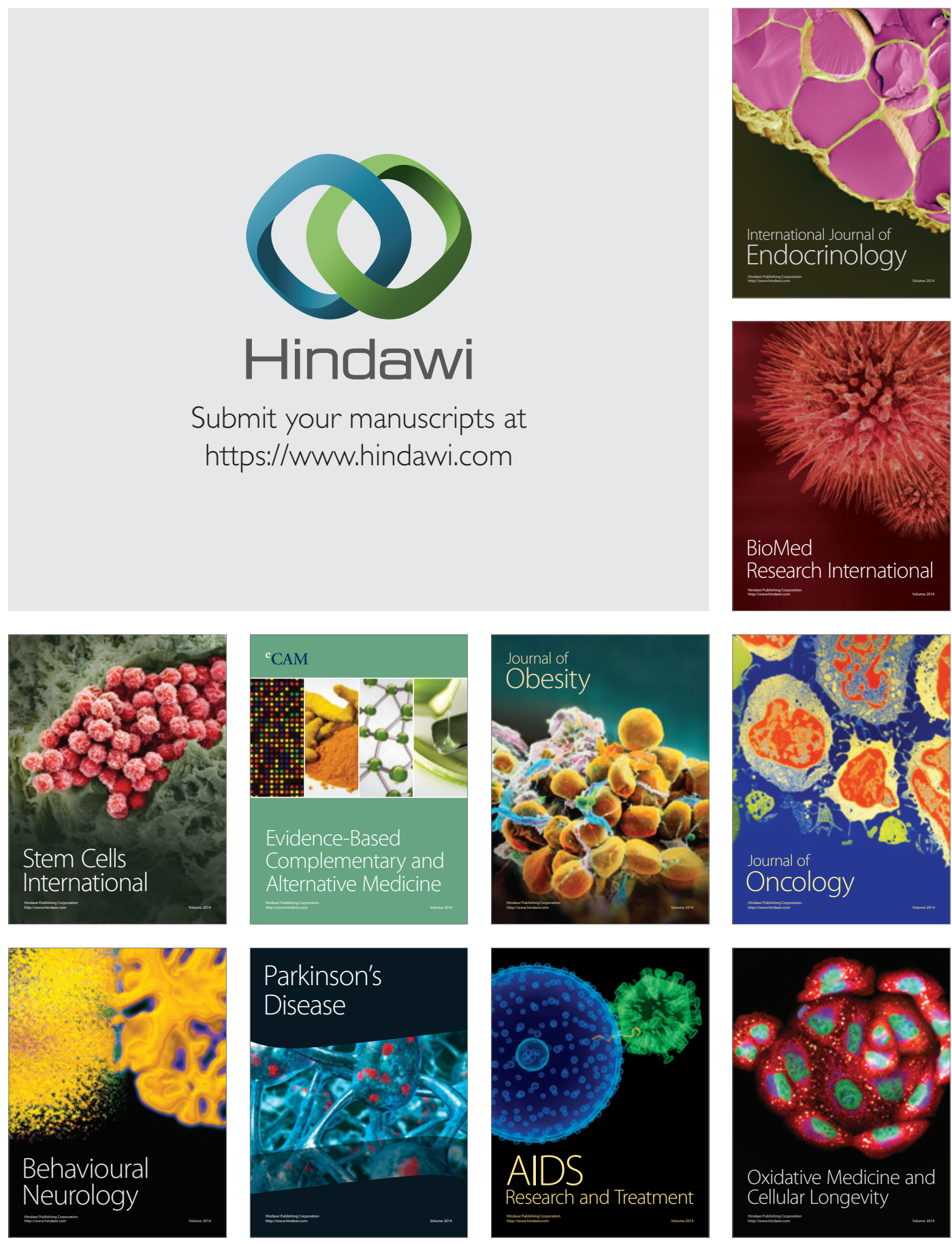